\title{
On the Growth of Ice-Crystals in the Air ${ }^{*}$
}

\author{
By K. Isono \\ Geophysical Institute, Tokyo University, Tokyo, Japan \\ (Manuscript received 31 May 1957)
}

\begin{abstract}
The processes of the ice-crystal formation on nuclei have been studied experimentally. Replicas of ice-crystals formed by seedings of $\mathrm{AgNO}_{3} \mathrm{MgO}, \mathrm{AgI}$, etc. were examined under optical and electron microscopes. The mode of the growth of ice-crystal by sublimation depends not only on temperature and water vapour excess, but also on condition of diffusion field of water vapor surrounding the crystal and impurities in air. The mode of ice-crystal growth is briefly discussed.
\end{abstract}

\section{Introduction}

The process of snow crystal formation in the atmosphere may be divided into the following two stages :

(1) Formation of a minute ice-crystal on an ice-forming nucleus or freezing of cloud droplets, and

(2) Growth of the minute ice crystal thus formed to a snow crystal of visible size by sublimation.

The first stage may be divided into substages of

(1) condensation of water vapour on a nucleus and

(2) freezing of the super-cooled droplet thus formed and formation of an ice-crystal. In the first stage, the nature of ice-form ing nuclei plays an important role. The results of electron microscope studies made by the author (Isono, 1955 and 1957b), Kumai (1951) and other investigators show that clay minerals and other soil particles whose atomic arrangement in their base planes is similar to that of the ice-crystal are the principal nuclei of snow crystals. Theoretical study made by Krastanow (1940) and experimental studies made by Fournier d'Albe (1949), Weickmann (1949) and Isono

\footnotetext{
* Division of Meteorology, Contribution No. 105.
}

(1957a and 1957b) show that the condensation of water vapour on nuclei at first occurs before the formation of ice-crystals.

In the second stage, ice-crystals grow by sublimation. The habit of the crystals depends on water vapour excess over ice saturation, temperature and the features of air flow surrounding the crystals.

In the present paper, some brief discussion about these two stages will be made.

\section{Formation of ice-crystals on nuclei}

As described in the previous paper (Isono, 1957a) nuclei insoluble in water such as AgI serve as ice-forming nuclei at humidities above or near water saturation, while hygroscopic nuclei such as $\mathrm{AgNO}_{3}$ serve as ice forming nuclei at humidities considerably below water saturation. It has been found (Isono, 1957b) that ice crystals were formed abundantly by spraying aqueous solution of $\mathrm{AgNO}_{3}$ into a cold chamber by means of a nebulizer, only when the concentration of $\mathrm{AgNO}_{3}$ was as low as the order of $0.001 \%$ in weight. The ice-crystals thus formed are shown in Fig. 1. In the figure, many ice crystals of nearly spherical shape can also be seen. When ice crystals formed by seeding of $\mathrm{AgI}$ or $\mathrm{AgNO}_{3}$ were sampled in a short time after the seeding by means of an impacter, 
75 th Anniversary Volume of the Journal of the Meteorological Society of Japan
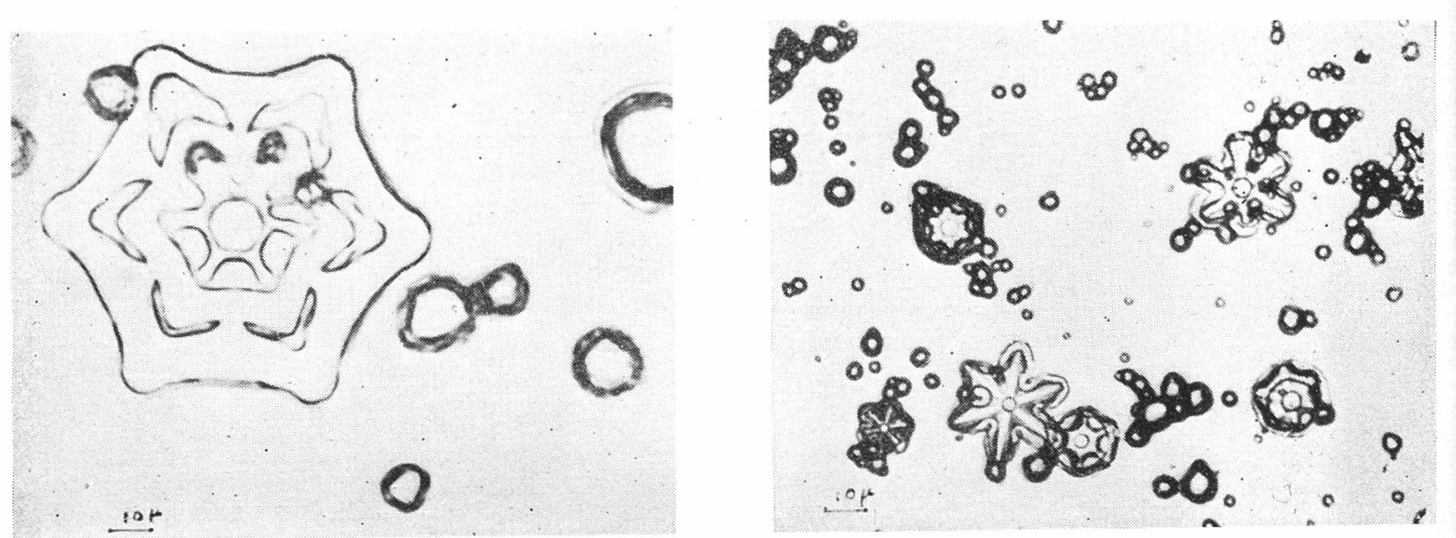

Fig. 1. Ice-crystals formed when aqueous solution of $\mathrm{AgNO}_{3}$ of the concentration of about $0.001 \%$ in weight was sprayed into a cold chamber by means of a nebulizer. $(\times 500)$

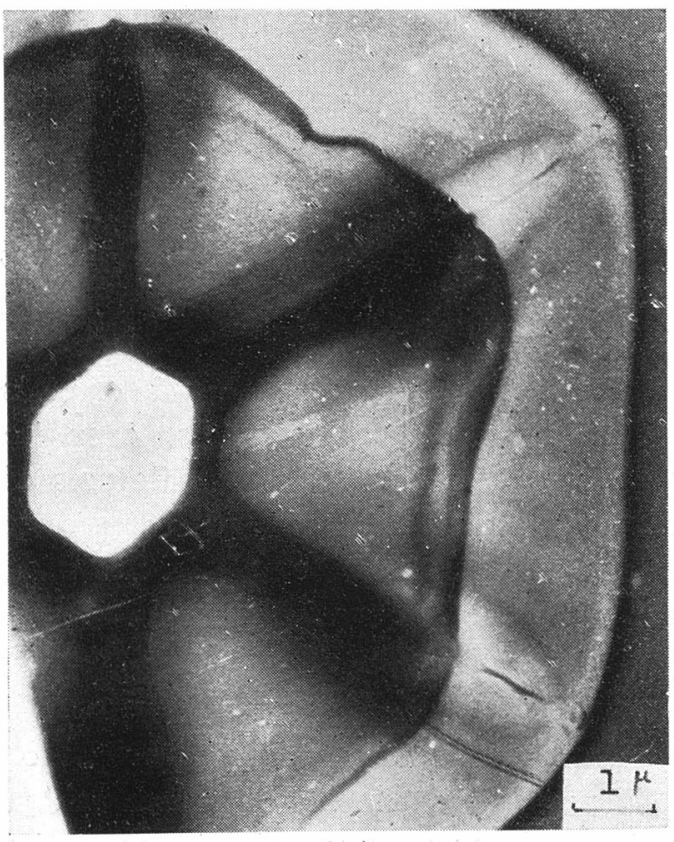

(a)

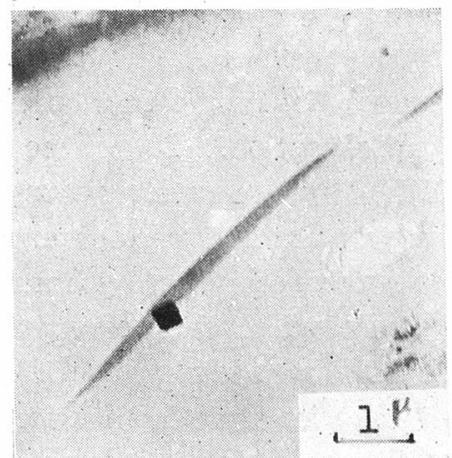

(c)

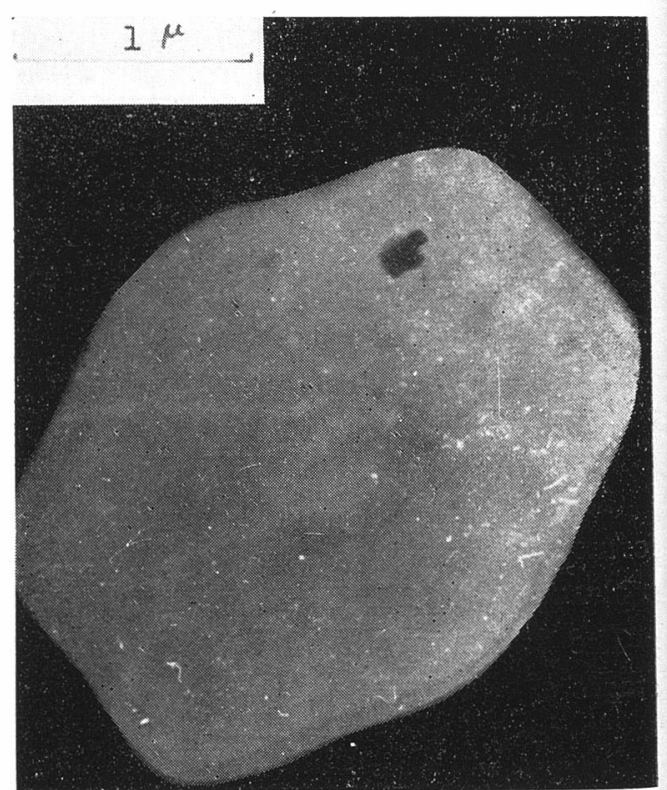

(b)

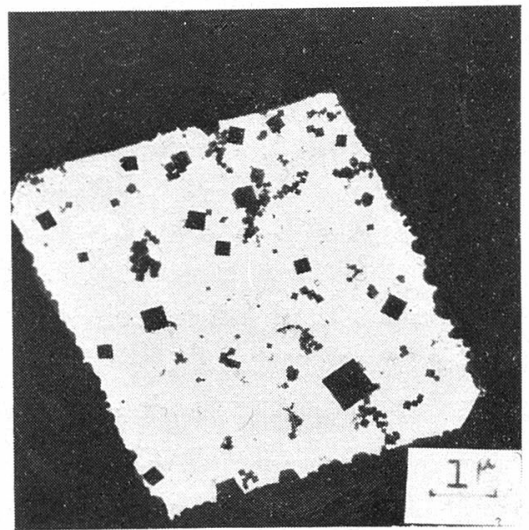

(a) and (b) are electron micrographs of an

Fig. 2. Ice-crystals formed by seeding of $\mathrm{MgO}$ smoke. ice-crystal of different magnifications. Cubic crystal of, $\mathrm{MgO}$ can be seen in the inner-most hexagon of the crystal. (c) is electron-micrograph of the inner-most hexagon of another ice-crystal in which a cubic crystal of $\mathrm{MgO}$ is seen. (d) Electron micrograph of magnesium oxide smoke particles used for seeding. $\mathrm{m}$

sho

an

res

ice

he

on

an

of

$-1$

pa

hex

as

pai

$\mathrm{Fr}$

the

Th

we

cry

be

the

cry

ser

$\operatorname{mic}$

nat

no

we

we

pat

mis

T

tha

seve

sequ

In $t$

ing

grov

of $t$

vali

the

0.00

size

3.

$\mathrm{Tl}$ been (Nal 
most of ice-crystal sampled were of spherical shape like "droxtals" found by Thuman and Robinson (1954) (Isono, 1957a). The result of electron microscope studies of ice crystal replicas shows that inner-most hexagons of ice crystals had dimensions of one to several microns in radius (Isono 1953 and 1957a). In Fg. 2 electron micrographs of ice-crystals formed by $\mathrm{MgO}$ seeding at $-16 \mathrm{C}$ water saturation are shown. The particles which are seen in the inner-most hexagons of the crystals can be identified as $\mathrm{MgO}$ crystals, when they are com pared with $\mathrm{MgO}$ crystals shown in Fig. 3. From the $\mathrm{MgO}$ particles, we could obtain the electron diffraction pattern of $\mathrm{MgO}$. The sites where these particles were found were in the inner-most hexagons of the crystals, but rot at their center. This may be perhaps partly due to the migration of the particles during sublimation of the icecrystals in formvar replicas. When we observe nuclei of ice-crystals with electron microscope, we should take care of contamination. In the case of nuclei which have no well-defined shape (for instance, $\mathrm{AgI}$ ), we can hardly identify their materials unless we can obtain their electron diffraction patterns. In such a case, one is apt to mistake other contaminants for nuclei.

The facts described above seem to show that super-cooled water droplets of one to several microns are formed at first and subsequent freezing of the droplets takes place. In the case of nuclei soluble in water, freezing occurs when super-cooled water droplets grow to a certain size and the concentration of the solution becomes lower than a specified value according to the nature of nuclei (in the case of $\mathrm{AgNO}_{3}$, the concentration is about $0.001 \%$ in weight, being estimated from the size of droxtal-like ice-crystals).

\section{Ice-crystal habit}

The modes of growth of ice-crystals have been studied by Nakaya and his collaborators (Nakaya 1954, Hanajima 1942, 1944) and others. Marshall and Langleben (1954) stated that the habit of ice-crystals depends on water vapour excess over ice saturation in ambient air. But, Shaw and Mason (1955) stated that the deciding factor of the crystal habit is not the vapour excess, but temperature. Generally speaking, the habit of a crystal is determined by relative velocities of growth of different faces of the crystal. The velocity of growth of a face depends on

(1) reaction rate, $\mathrm{k}$, at which molecules are arranged in the lattice of the crystal,

(2) diffusion field around the crystal and saturation vapour pressure at the face, and

(3) rate of transfer of heat generated by sublimation of vapour on the crystal.

When one of these conditions varies. the crystal will grow in different mode. Although water vapour excess and temperature in ambient air may be principal factors on which the habit of ice crystals depends, the habit depends also upon factors which have influence on the conditions (2) and (3); features of air flow arround the crystal, the method of support of the ice-crystal and cooling rate. Adsorption of impurities on a face will change the $\mathrm{k}$ of the face. The effect of the reaction rate $\mathrm{k}$ at the crystal faces and the thickness of the boundary layer has been discussed elsewhere (Isono, 1957b). When the thickness of the boundary layer surounding the crystal is small or reaction rate is small compared with molecular diffusion coefficient D of water vapour in air, the growth of the ice-crystal is governed not only by the diffusion field around the crystal but also by reaction rate $\mathrm{k}$. It might be expected that ice crystals might grow in different mode under the condition of different value of $D$; for instance, in hydrogen atmosphere or under low pressure, such a condition may be realised

The well-known equation of the ice-crystal growth and the ice-crystal models pressented by Houghton (1950) are very useful and convenient for the calculation of the growth 
rate but, stricktly speaking, the growth rate obtained from experiments (Isono et al, 1956 and 1957) does not agree with the calculated values from the equation. This seems to be due to the circumstances that effects of the reaction rate and the boundary layer are not taken into account in the equation except for velocity coefficient and that the models of ice-crystals are not satisfactory for accurate calculation. It is necessary to adopt models more simulated to real ice-crystals than those adopted by Houghton and to take account of the effect of turbulence in order to calculate the growth rate accurately.

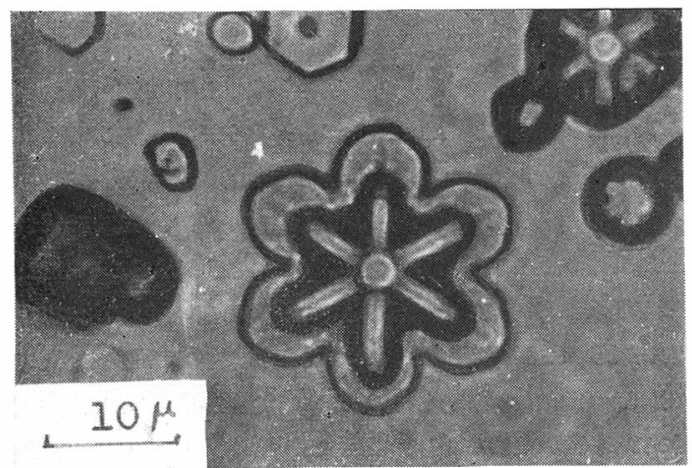

Fig. 3. Ice-crystals formed at $-16^{\circ} \mathrm{C}$, water saturation. photomicrograph of the same crystals shown in (a). be determined from the figures.

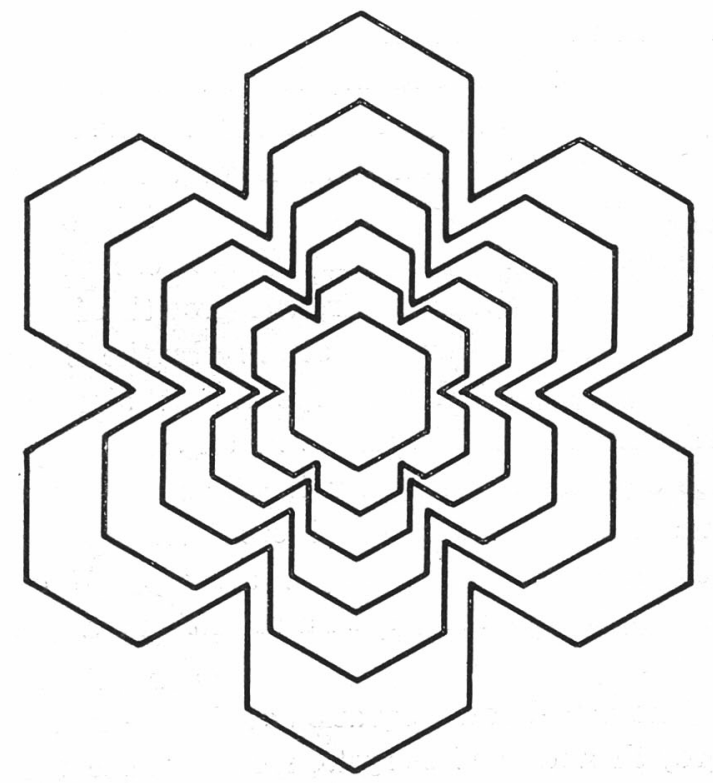

Fig. 4. Sketch of an ice-crystal seen at the center of fig. 3a. lowest at the center.
But, in general, we cannot calculate the diffusion fields analytically for such complicated models. Numerical calculation will yield good result.

In the case of the actual ice-crystal, growth at edges of the crystal plays an important role in the growth of the crystal. In Fig. 3 , hexagonal plates and stellar ice-crystals formed at $-16 \mathrm{C}$ with water saturation are shown; (b) is the phase-contrast photomicrograph of the same crystals as those shown in (a). A sketch of the ice-crystal seen at the center of Fig. 4 is shown in Fig. 4. This figure tells us the mode of growth of

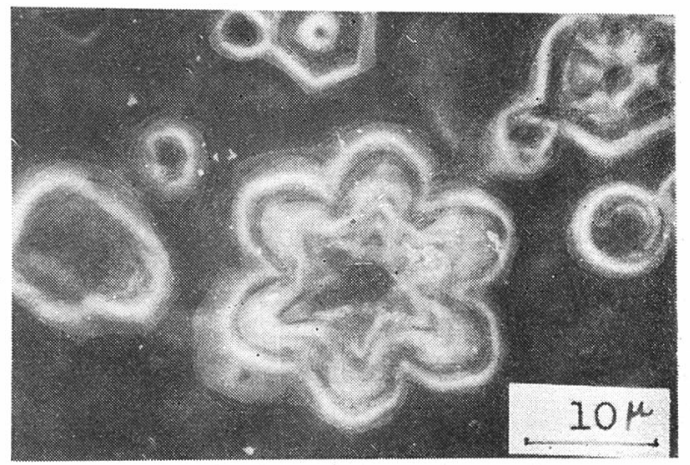

(b) is the phase-contrast Height contour lines can the ice-crystal. As the flux density of water vapour at each edge is large, the growth rate is large near each edge. Consequently, extension occurs at each edge as shown in Fig. 5a. For instance, at the edge A

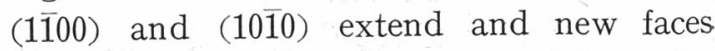
$(0 \mathrm{~h} \overline{\mathrm{h}} 0)$ and $(0 \overline{\mathrm{h}} \mathrm{h} 0)$ appear. When the crystal growth is principally controlled by diffusion field and vapour excess in ambient air is large, the growth rates of (1100) and (1010) are large. In this case. an ice-crystal of stellar shape or plate with broad branches as shown in Fig. $5 \mathrm{~b}$ will result.

When the crystal growth is controlled not only by the diffusion field, but also by the reaction rate at the surface or when the water vapour excess is small, the velocities of extension of (1100) and (1010) at the edge $\mathrm{A}$ are small and the positions where reaction 
On the Growth of Ice-Crystals in the Air

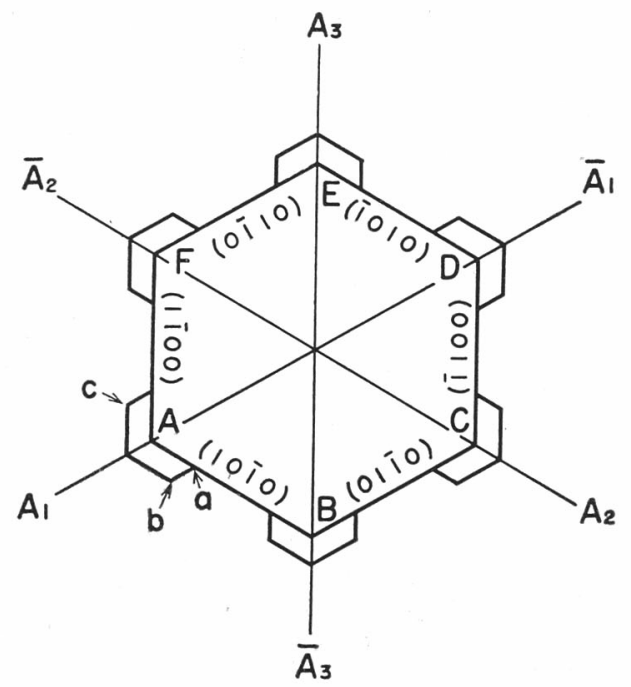

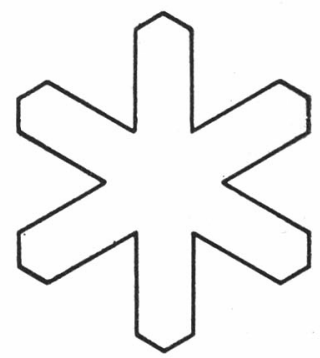

(b)

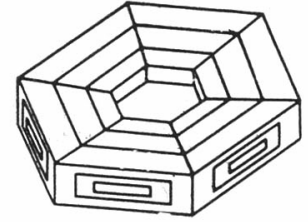

(c)

(a)

Fig. 5. Mode of growth of ice-crystal. c: lowest at the center.

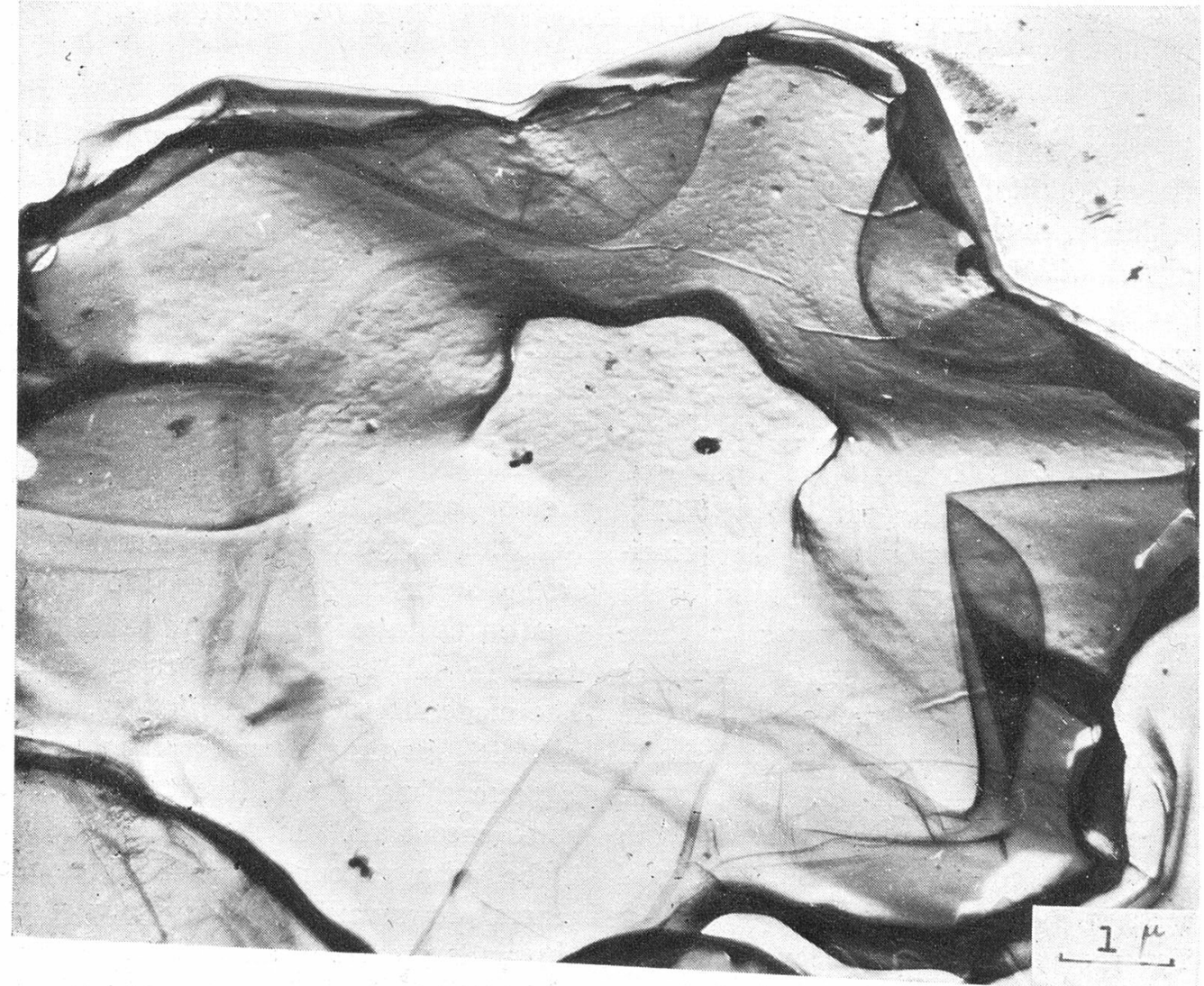

Fig. 6. Electron micrograph of ice-crystal replica (carbon film). Highest the inner-most hexagon; lowest the corresponding innermost hexagon of the ice-crystal. 


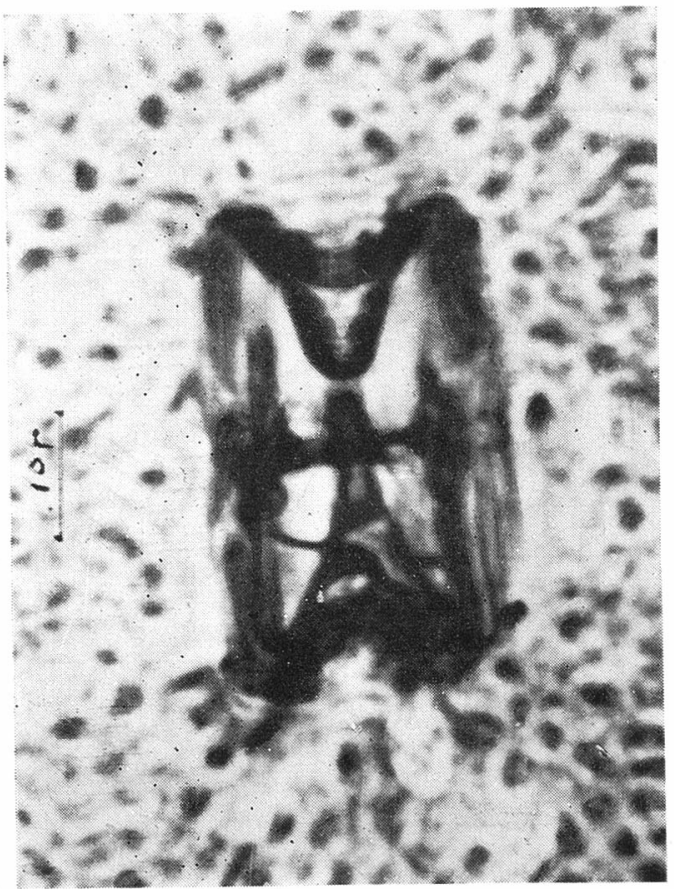

( a )

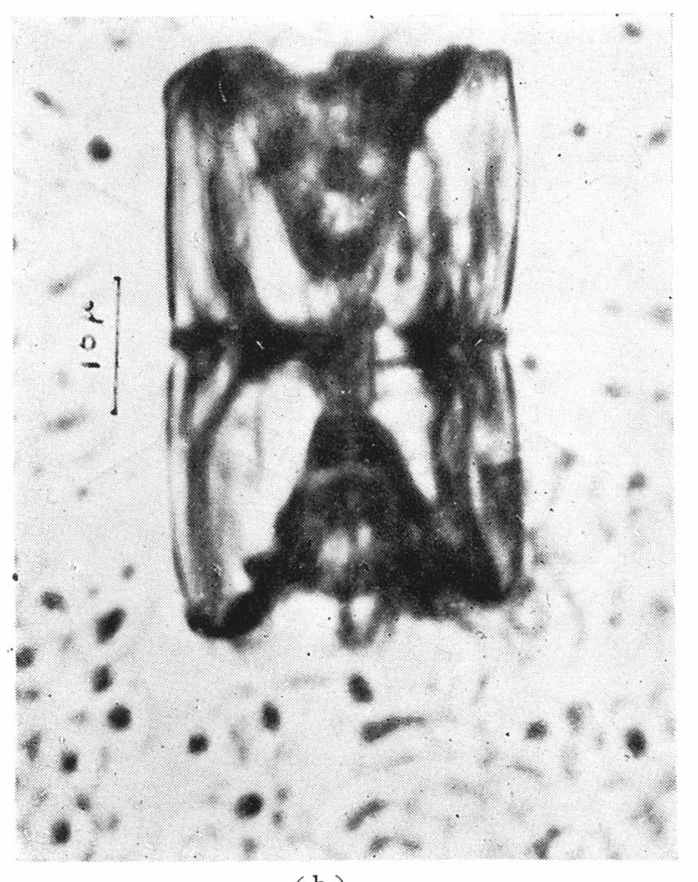

(b)

Fig. 7. Typycal examples of columnar ice-crystals which show growth at edges. Dent on face of prism can be seen.

rate is large appear and the velocities of growth of $(0 \mathrm{~h} \overline{\mathrm{h}} 0)$ and $(0 \overline{\mathrm{h}} \mathrm{h} 0)$ at $\mathrm{A}$ are comparable with the former. Consequently, the crystal will grow to a large hexagonal plate as shown in Fig. 5c.

The mode of growth of columnar icecrystals may be explained in a similar way. In this case, growth rate at the edges parallel to C-axis is small, as compared with that at the six edges parallel to (0001). The growth at the latter plays the principal role. When the effect of diffusion field is predominant, the growth rates at these edges are large and the crystel will grow as shown in Fig. $7 \mathrm{~b}$. At the center of the each face of the form [1010], a dent is commonly found (c.f. Fig. 7). This is due to the greater activity or the greater growth velocity at the edge than at the centers of the faces.

When the vapour excess is large and the growth is governed almost only by diffusion field, branches will develop at newly formed edges or corners (such as b or c in Fig. 6) and the crystals will grow to dendrites.

As described before, the ice-crystal growth is controlled by the diffusion field around the crystal, so we should take these effects into account in the interpretation of the result of an experiment on the habit and the growth rate of ice-crystals. When icecrystals are formed in thermodynamically unstable air current as was in the case of Nakaya's experiment, the boundary layer around ice crystals is considered to be thinner than in the case where the experiment is made in still air in a diffusion chamber. The mode of growth of an ice-crystal in air in which gradient of water vapour density is present may be different from that in homogeneous air. In the case of the former, if the water vapour density is higher above, general downward diffusion flow of water vapour is present in air and the ice-crystal will grow on its upper surface, while on its lower surface growth rate may be small and 
even evaporation may take place. These circumstance will bring forth particular modes of growth. Therefore, we should be very careful about the conditions under which ice-crystal grow.

It is conceivable that shapes of natural snow crystals depend not only on air temperature and humidity at which they grow, but also on the condition of turbulence in clouds in which they grow; it might be expected that shapes of ice-crystals formed in a stratus-type cloud might differ from those formed in a cumulus-type cloud of the same temperature and humidity.

\section{Conclusion}

The experimental results described in $\$ 2$ may be considered to show that condensation of water vapour on nuclei takes place in the preceding stage to the formation of ice-crystal and water droplets of one to several microns are formed and subsequent freezing occurs. The growth of an ice crystal thus formed is considered to be controlled by the reaction rate at the crystal faces, temperature and water vapour density in ambient air, the condition of air flow (turbulence) surrounding the crystal and impurities in air. As the growth at the crystal edges plays an important role in ice-crystal growth process as described in $\$ 3$, we should adopt models, in which these effect are taken into account for the calculation of growth rate of the icecrystal.

\section{Acknowledgement}

The author wishes to express his sincere thanks to Mr. Yotsumoto and other members of the Japan Electron-Optical Laboratory Co. Ltd. to whom he is indebted in taking the electron micrographs.

\section{References}

1) Fournier d'Albe, E. M. 1949: Some experiments on the condensation of water vapour at tmperature below $0{ }^{\circ}$ C. Quart. J. r. meteor. Soc. 75 1-14. -2) Hanajima, M. 1942: On the condition for the formation of snow crystals. (in Japanese) J. meteor. Soc. Japan Ser II 20 238-251. -3) - 1944: Supplementary to "On the condition for the formation of snow crystals." (in Japanese) J. meteor. Soc. Japan Ser II 22 123-127. -4) Houghton, H. G., 1950: A preliminary quantitative analysis of precipitation mechanism. J. meteor. 7 363-369. -5) Isono, K. 1953: An electron microscope study of ice crystal formation. J. meteor. Soc. Japan. Ser II 31 318-322. -6) - 1955: On ice-crystal nuclei and other substances found in snow crystals. J. Meteor. 12 456-462. -7) - 1957a: On iceforming nuclei. J. meteor. Soc. Japan. Ser II 35 67-70. -8) — 1957b: Microphysical processes in precipitation mechanism. (To be published). -9) Isono, K., M. Komabayasi, Y. Yamanaka and H. Fujita, 1956: An experimental investigation of the growth of ice-crystals in a super-cooled cloud. J. meteor. Soc. Japan. Sea II 34 158-163. -10) Isono, K., M. Komabayasi and A. Ono, 1957: On the growth rate of ice-crystals in super-cooled steam fog. (in Japanese) Report of Studies on Artificial Rain-making. Tokyo Electric Power Co. Ltd., Geophys. Inst., Tokyo Univ. June 1957 - 11) Krastanow, L., 1940: Über die Bildung der unterkühlten Wassertropfen und die Eiskristalle in der freien Atmosphäre Met. Zeit. 57 357-371. -12) Kumai, M., 1951: Electron microscope study of snow-crystal nuclei $J$. Meteor. 8 151-156. -13) Marshall, J. S. and M. P. Langleben, 1954: A theory of snow'crystal habit and growth.J. Meteor 11 104-120. -14) Nakaya, U., 1954: Snow Crystals. Natural and artificial. Harvard University Press. -15) Shaw, D. and B. J. Mason, 1955: The growth of ice-crystals from the vapour. Phil. Mag. Ser. 7, 46 249-262. -16) Thuman W. C. and E. Robinson, 1954: Studies of Alaskian ice-fog particles. J. Meteor. 11 151-156. -17) Weickmann, H. 1949: Die Eisphase in der Atmosphäer. Ber. Dt. Wetterd. U. S.-Zone Nr. 6. 